INDUSTRIA Y TECNOLOGÍA

\title{
Análisis de la afectación causada por el deterioro de la malla vial del tramo de la estación de Transmilenio-Escuela Militar de la calzada occidente-oriente
}

\author{
Johan Camilo Rodríguez Cerón \\ Sergio Esteban Valero Hernández \\ Javier Alejandro Villota Arteaga \\ Octavio Vega \\ Johanna Carolina Ruiz Acero
}

Escuela Militar de Cadetes "General José María Córdova”

\begin{abstract}
RESUMEN
El deterioro del tramo de la malla vial de la Estación Escuela Militar de Transmilenio (ET-ESMIC) impacta negativamente la infraestructura de la estación, el parque automotor y la población usuaria del Sistema Integrado de Transporte Transmilenio (SITT). En este trabajo se estima el nivel de deterioro de la malla vial del tramo de la ET-ESMIC de acuerdo con la tipología de daños que se evidencian en el tipo de pavimento de la calzada. Para esto se estudia la velocidad de los articulados al transitar en el tramo de la malla vial deteriorada, específicamente en la afectación a las variables de tiempo y comodidad, además se mide el Índice de Rugosidad Internacional (IRI), aplicando métodos que comparativamente permitan obtener la mayor precisión en el resultado de la variable en estudio. Finalmente, con base en los resultados obtenidos se recomienda aplicar el Manual de Pavimentos Flexibles para hacer una inspección debidamente respaldada en los lineamientos del Instituto Nacional de Vías (Invias).
\end{abstract}

\section{PALABRAS CLAVE}

Índice de Rugosidad Internacional (IRI); pavimentos flexibles; rehabilitación vial; Sistema Integrado de Transporte Transmilenio; vehículos articulados.

\section{CITACIÓN}

Rodríguez Cerón, J. C., Valero Hernández, S. E., Villota Arteaga, J. A., Vega, O., \& Ruiz Acero. J. C. (2019). Análisis de la afectación causada por el deterioro de la malla vial del tramo de la estación de Transmilenio-Escuela Militar de la calzada occidente-oriente. Revista Brújula de Investigación, 7(14), 17-25. https://doi.org/10.21830/ 23460628.20 


\section{Introducción}

La Calle 80 y otras vertientes vehiculares habilitadas para la instalación y funcionamiento del Sistema Integrado de Transporte Transmilenio (SITT) han sufrido deterioro de la malla vial, lo cual ha afectado tanto la infraestructura del sistema, como también el parque automotor y la población involucrada (conductor, usuarios y vecindad).

La troncal de la Calle 80 se empezó a construir desde 1970, con una estructura de pavimento flexible. La firma Steer, Davis y Gleave Ltda. realizó el diseño geométrico de la vía, buscando la adaptación al sistema Solo-Bus. El Consorcio Restrepo y Uribe Ltda.-Sesac Ltda. diseñó la estructura del pavimento flexible para una vida útil de quince años. Su construcción se contrató con ICA de México (figura 1). En las primeras etapas de la obra se optó por integrarse a Transmilenio, pero por las diferencias de cargas con el Solo-Bus, su vida de diseño se redujo a siete años. Cuando ICA de México la abandonó había construido cerca del 60\% de la obra. (Beltrán, 2006).

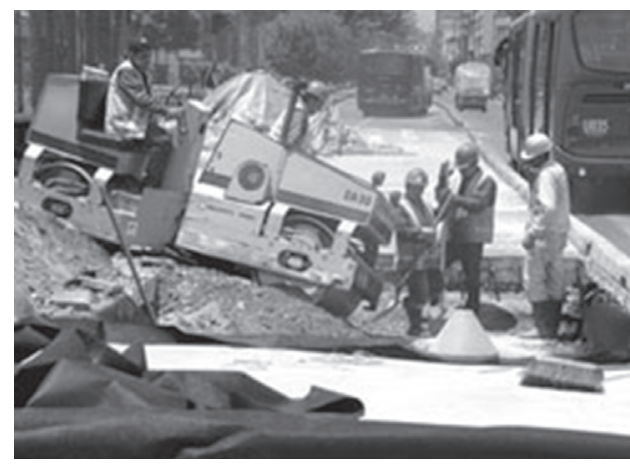

Figura 1. Los buses de Transmilenio soportan una carga de hasta 12,5 toneladas

Fuente: Bernal (2006).

En cuanto a los conceptos técnicos de la obra, se utilizó un asfalto modificado con polímeros en la conformación de la capa de roda- dura de las calzadas de TM, lo cual significó en ese momento una innovación en el uso de nuevos materiales en el país. Se usó con el fin de aumentar la vida de diseño del pavimento y de disminuir la deformación viscosa que experimentan las mezclas asfálticas bajo las cargas repetidas. Se tienen datos que en septiembre de 2003 las fallas afectaban cerca del $32 \%$ de la superficie del pavimento en las calzadas de TM, y del $14 \%$ en las calzadas de tráfico mixto (Beltrán, 2016).

\section{Metodología}

El tramo específicamente seleccionado para el presente estudio tiene una extensión de 180 metros y abarca la Estación de Transmilenio-Escuela Militar, designada para el tránsito de los articulados del Sistema Integrado de Transporte Masivo-Transmilenio.

Después de delimitar el tramo, se desarrollaron tres fases metodológicas de estudio y un enfoque mixto (cualitativo y cuantitativo), acompañado de una trazabilidad para obtener los datos estadísticos y la información necesaria que permita hacer un análisis de la problemática y los resultados de actividades que llevaran a obtener aportes para fundamentar las soluciones y que posibiliten que se conviertan en prototipo para replicar en otros tramos del SITT y optimizar las estaciones que todos los ciudadanos necesitan.

El contexto del estudio es el tramo sobre la extensión que comprende la calzada de la ET-ESMIC en razón de ser una de las transitadas por el SITT, razón por la cual se presentan más deterioros por el peso que tienen cuando circulan. La caracterización del contexto se realizó a través de visitas a campo y selección de puntos con deterioro. En la ET-ESMIC se logró encontrar un tramo acorde con las necesidades del estudio: el tramo en sentido occidente-oriente 
(figura 2, en amarillo) se encontraba en un alto estado de deterioro, de manera que se pudo realizar el levantamiento topográfico y calcular el Índice de Rugosidad Internacional (IRI), tomar la reducción de velocidad de marcha y la afectación al parque automotriz, a la infraestructura de la malla vial y a la población involucrada (usuario, conductor y vecindad), como se muestra en la figura 2.

\section{Creación de perfiles longitudinales de la superficie}

A partir de la superficie obtenida en el levantamiento topográfico se trazaron cuatro perfiles longitudinales, dos a cada lado de la calzada por donde se estima pisan las llantas al transitar el daño en el tramo, y se simuló el movimiento vertical del bus. Para la creación de los perfiles se exagera el eje de las cotas en $10 \%$. A continuación, se muestran los perfiles seleccionados (figuras 3, 4, 5 y 6).

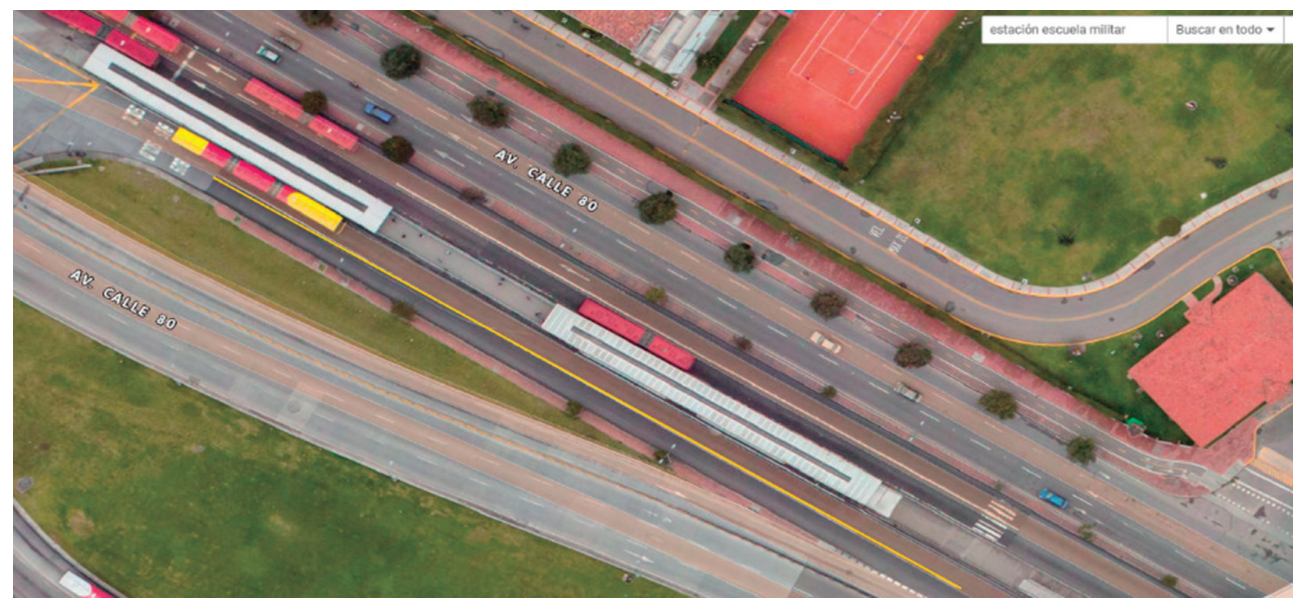

Figura 2. Localización tramo en estudio

Fuente: Mapas Bogotá. Google Maps.

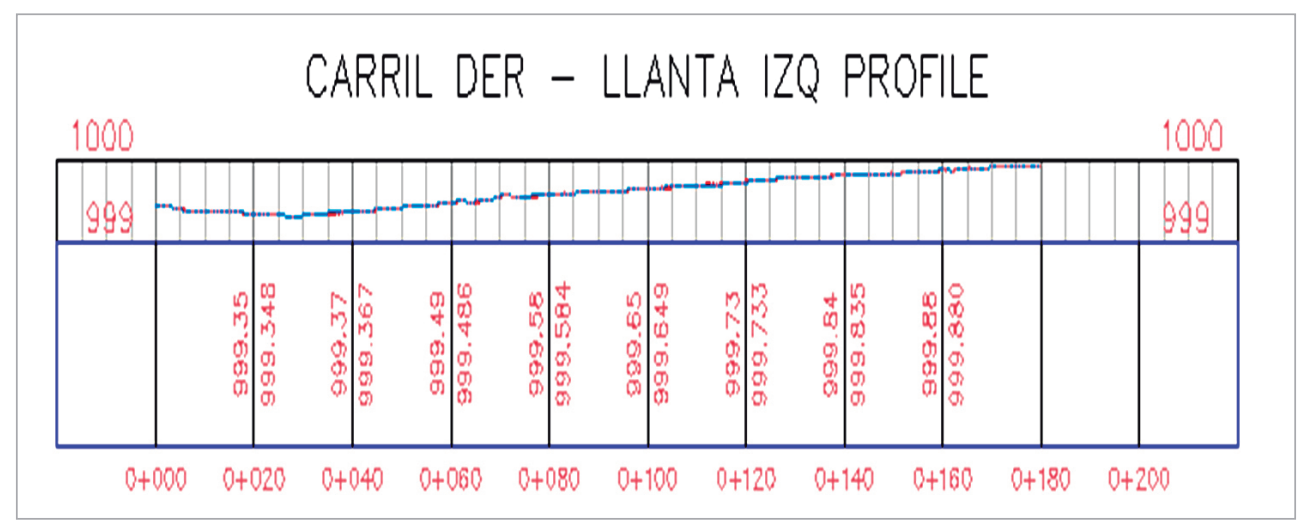

Figura 3. Perfil 1: carril derecho borde interno

Fuente: elaboración propia. 


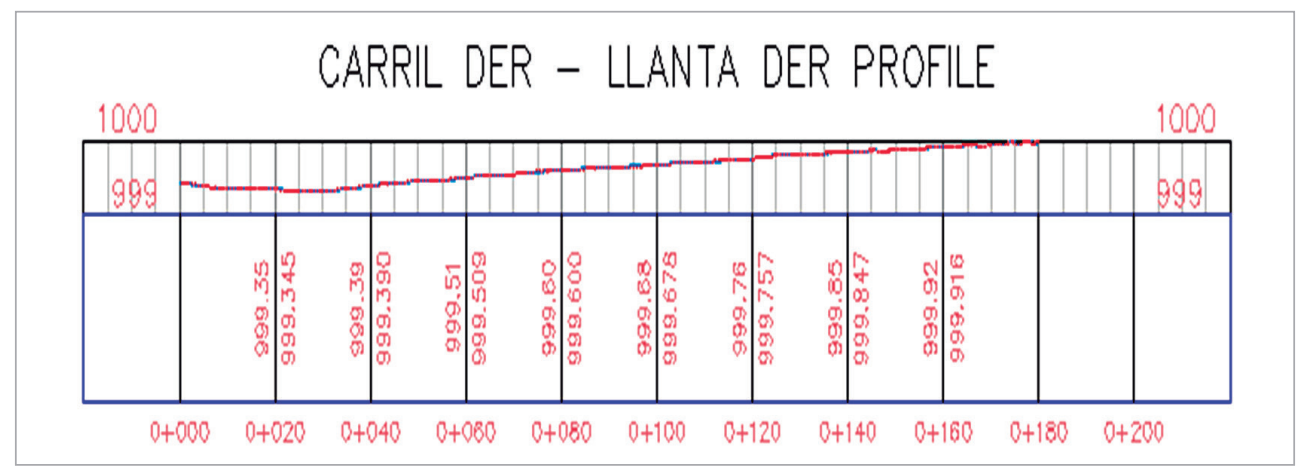

Figura 4. Perfil 2: carril derecho borde externo

Fuente: elaboración propia.

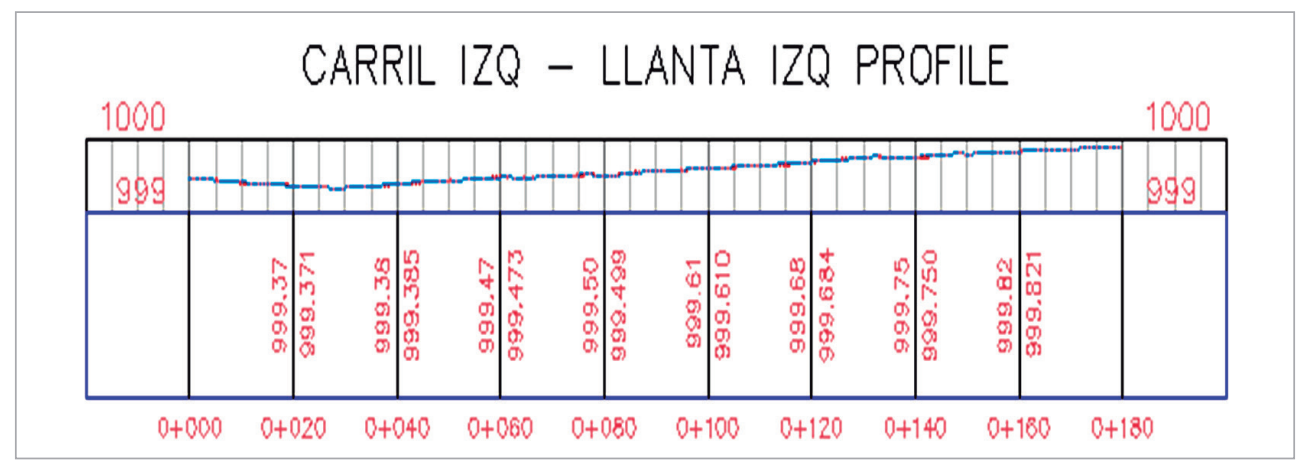

Figura 5. Perfil 3: carril izquierdo borde interno

Fuente: elaboración propia.

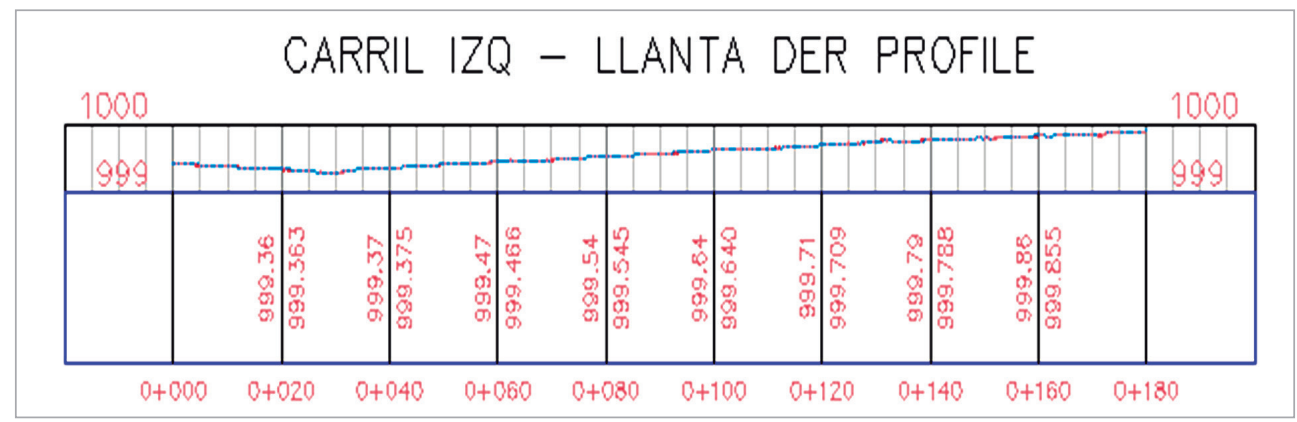

Figura 6. Perfil 4: carril izquierdo borde externo

Fuente: elaboración propia.

\section{Resultados}

El tramo estudiado tiene un área de 1258,54 $\mathrm{m}^{2}$ y evidencia un área afectada de $289.925 \mathrm{~m} 2$, equivalente al 23,04 \% del tramo. En la tabla 1 se muestra el resumen de inspección visual realizada, que incluye las áreas afectadas por severidad y tipo de daños: ahuellamiento (AHU), fisura longitu- 
dinal (FL), descascaramiento (DC), piel de cocodrilo (PC), pulimento del agregado (PU) y cabezas duras (CD), fisura en bloque (FB), fisura transversal (FT), fisuración incipiente (FIN), pérdida del agregado (PA), bache (BCH) y desgaste superficial (DSU).

Tabla 1. Formato del Manual de Inspección Visual de Pavimentos Flexibles (MIV-PF) para informe de inspección visual

\begin{tabular}{|c|c|c|c|c|c|c|c|c|c|c|c|c|c|}
\hline \multirow{2}{*}{ Tramo } & \multirow{2}{*}{$\begin{array}{l}\text { Ancho } \\
(\mathrm{m})\end{array}$} & \multicolumn{2}{|c|}{ Abscisa } & \multirow{2}{*}{$\begin{array}{c}\text { Área tramo } \\
\left(\mathrm{m}^{2}\right)\end{array}$} & \multicolumn{3}{|c|}{$\mathrm{AHU}$} & \multicolumn{3}{|c|}{$\mathrm{BCH}$} & \multicolumn{3}{|c|}{ DC } \\
\hline & & Desde & hasta & & B & $\mathrm{M}$ & A & B & $\mathrm{M}$ & A & B & $\mathrm{M}$ & A \\
\hline 1 & 7 & $\mathrm{~K} 0+000.000$ & K0+179.792 & 1.258 .544 & 51,88 & 0 & 0 & 0 & 0 & 0,205 & 0,175 & 0 & 0,683 \\
\hline \multicolumn{4}{|c|}{ Área total inspeccionada (m²) } & 1.258 .544 & & & & & & & & & \\
\hline \multicolumn{5}{|c|}{ Área total afectada por severidad $\left(\mathrm{m}^{2}\right)$} & 51,88 & 0 & 0 & 0 & 0 & 0,205 & 0,175 & 0 & 0,683 \\
\hline \multicolumn{5}{|c|}{ Área total afectada por tipo de daño $\left(\mathrm{m}^{2}\right)$} & \multicolumn{3}{|c|}{51,88} & \multicolumn{3}{|c|}{0,205} & \multicolumn{3}{|c|}{0,858} \\
\hline \multicolumn{5}{|c|}{ Peso del daño por severidad dentro del área inspeccionada } & 4,122 & 0 & 0 & 0 & 0 & 0,016 & 0,014 & 0 & 0,054 \\
\hline \multicolumn{5}{|c|}{ Peso total por tipo de daño del área inspeccionada (\%) } & \multicolumn{3}{|c|}{4,122} & \multicolumn{3}{|c|}{0,016} & \multicolumn{3}{|c|}{0,068} \\
\hline
\end{tabular}

\begin{tabular}{|c|c|c|c|c|c|c|c|c|c|c|c|c|c|}
\hline \multirow{2}{*}{ Tramo } & \multirow{2}{*}{$\begin{array}{l}\text { Ancho } \\
\text { (m) }\end{array}$} & \multicolumn{2}{|c|}{ Abscisa } & \multirow{2}{*}{$\begin{array}{l}\text { Área tramo } \\
\left(\mathrm{m}^{2}\right)\end{array}$} & \multicolumn{3}{|c|}{ DSU } & \multicolumn{3}{|c|}{$\mathrm{FB}$} & \multicolumn{3}{|c|}{ FCT } \\
\hline & & Desde & hasta & & B & $\mathrm{M}$ & A & B & $\mathrm{M}$ & A & B & $\mathrm{M}$ & A \\
\hline 1 & 7 & $\mathrm{~K} 0+000.000$ & K0+179.792 & 1.258 .544 & 3,141 & 0 & 0 & 6,862 & 8,753 & 0 & 0,287 & 0 & 0 \\
\hline \multicolumn{4}{|c|}{ Área total inspeccionada $\left(\mathrm{m}^{2}\right)$} & 1.258 .544 & & & & & & & & & \\
\hline \multicolumn{5}{|c|}{ Área total afectada por severidad $\left(\mathrm{m}^{2}\right)$} & 3,141 & 0 & 0 & 6,862 & 8,753 & 0 & 0,287 & 0 & 0 \\
\hline \multicolumn{5}{|c|}{ Área total afectada por tipo de daño $\left(\mathrm{m}^{2}\right)$} & \multicolumn{3}{|c|}{3,141} & \multicolumn{3}{|c|}{15,615} & \multicolumn{3}{|c|}{0,287} \\
\hline \multicolumn{5}{|c|}{ Peso del daño por severidad dentro del área inspeccionada } & 0,25 & 0 & 0 & 0,545 & 0,695 & 0 & 0,023 & 0 & 0 \\
\hline \multicolumn{5}{|c|}{ Peso total por tipo de daño del área inspeccionada (\%) } & \multicolumn{3}{|c|}{0,25} & \multicolumn{3}{|c|}{1,241} & \multicolumn{3}{|c|}{0,023} \\
\hline
\end{tabular}

Fuente: elaboración propia.

\section{Tipificación de los daños en el tramo de la malla vial de la ET-ESMIC según su severidad}

Las patologías de severidad baja en el tramo de la malla vial de la ET-ESMIC se muestran en la figura 7.

\begin{tabular}{|c|c|c|}
\hline \multicolumn{3}{|c|}{$\begin{array}{l}\text { DISTRIBUCIÓN DE DAÑOS DE } \\
\text { SEVERIDAD BAJA POR TIPO }\end{array}$} \\
\hline $\begin{array}{l}\text { TIPO DE } \\
\text { DAÑO }\end{array}$ & $\begin{array}{c}\text { ÁREA } \\
\text { AFECTADA } \\
\left(\mathrm{M}^{2}\right)\end{array}$ & $\begin{array}{c}\% \\
\text { DAÑO }\end{array}$ \\
\hline AHU & 51,88 & 19 \\
\hline DC & 0,175 & 0,06 \\
\hline DSU & 3,141 & 1,15 \\
\hline FB & 6,862 & 2,51 \\
\hline FCT & 0,287 & 0,11 \\
\hline FL & 8,89 & 3,26 \\
\hline FT & 0,584 & 0,21 \\
\hline PC & 179,711 & 65,82 \\
\hline $\mathrm{PCH}$ & 21,511 & 7,88 \\
\hline TOTAL & 273,041 & 100 \\
\hline
\end{tabular}

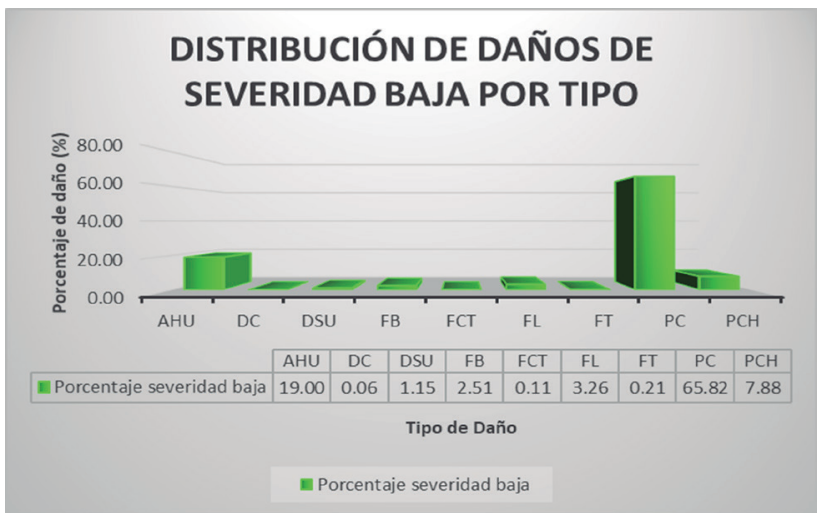

Figura 7. Distribución de severidad baja por tipo de daño en la ET-ESMIC Fuente: Ruiz et al. (2019). 
Las patologías de severidad media en el tramo de la malla vial de la ET-ESMIC se muestran en la figura 8.

\begin{tabular}{ccc}
\hline \multicolumn{3}{|c}{ DISTRIBUCIÓN DE DAÑOS DE SEVERIDAD } \\
BAJA POR TIPO \\
\hline $\begin{array}{c}\text { TIPO DE } \\
\text { DAÑO }\end{array}$ & $\begin{array}{c}\text { ÁREA } \\
\text { AFECTADA }\left(\mathrm{m}^{2}\right)\end{array}$ & \% DAÑO \\
\hline FB & 8,753 & 53,33 \\
\hline PA & 1,111 & 6,77 \\
\hline PC & 6,548 & 39,9 \\
\hline TOTAL & 16,412 & 100 \\
\hline
\end{tabular}

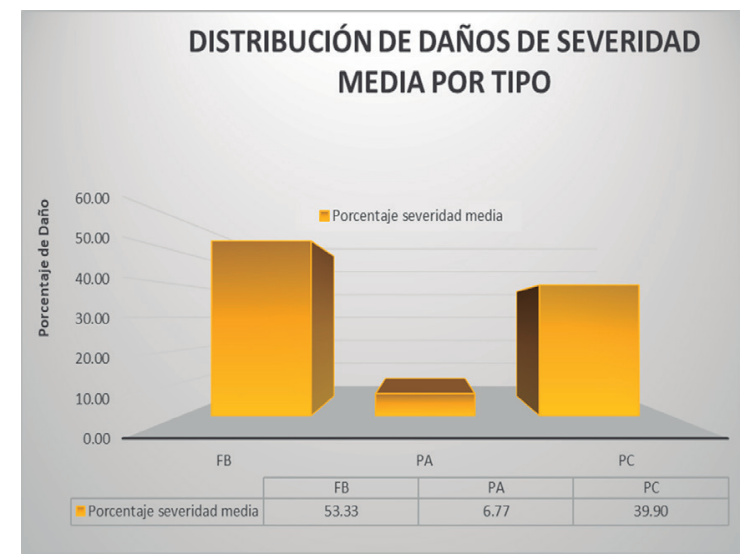

Figura 8. Distribución de severidad media por tipo de daño en la ET-ESMIC

Fuente: Ruiz et al. (2019).

Las patologías de severidad alta en el tramo de la malla vial de la ET-ESMIC se muestran en la figura 9.

\begin{tabular}{ccc}
\hline \multicolumn{3}{|c|}{ DISTRIBUCIÓN DE DAÑOS DE SEVERIDAD } \\
ALTA POR TIPO \\
\hline $\begin{array}{c}\text { TIPO DE } \\
\text { DAÑO }\end{array}$ & $\begin{array}{c}\text { ÁREA } \\
\text { AFECTADA }\left(\mathrm{m}^{2}\right)\end{array}$ & $\%$ DAÑO \\
\hline DC & 1,49 & 87,91 \\
\hline BCH & 0,205 & 12,09 \\
\hline TOTAL & 1,695 & 100 \\
\hline
\end{tabular}

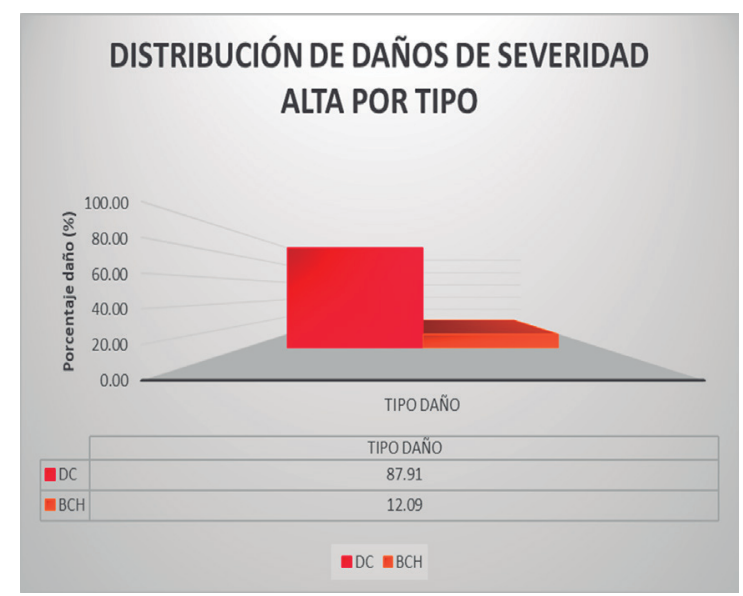

Figura 9. Distribución de severidad alta por tipo de daño en la ET-ESMIC

Fuente: Ruiz et al. (2019).

\section{Toma de velocidades de los buses articulados}

Los resultados de la toma de velocidades en el tramo de la ET-ESMIC en la reducción de velocidad al paso del articulado sobre el daño en pavimento por día se muestra en la figura 10. 


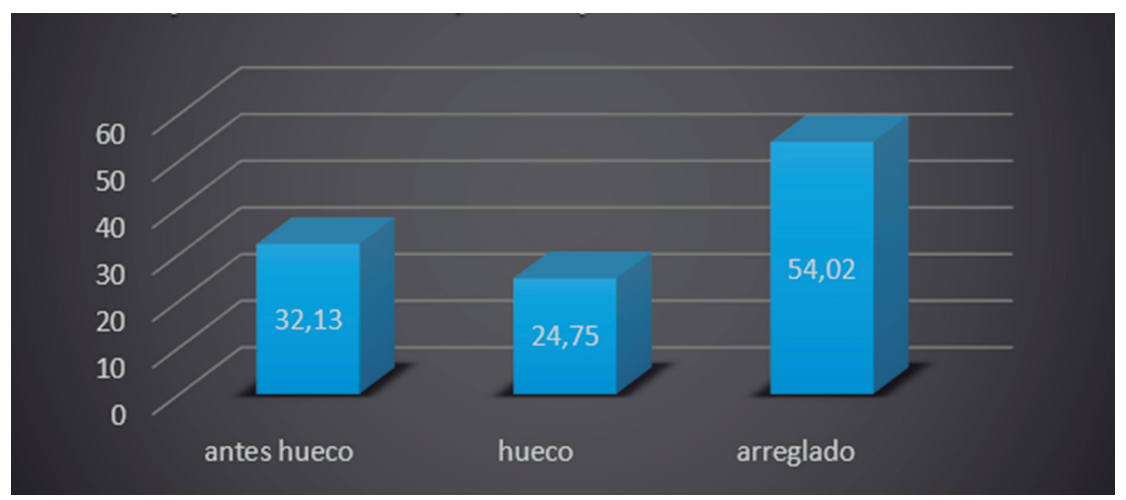

Figura 10. Resultados de la toma de velocidad por día en el tramo con daño en pavimento ET-ESMIC Fuente: elaboración propia.

\section{Análisis de resultados}

El daño que más se presenta corresponde al grupo de fisura denominada piel de cocodrilo (tabla 2).

Tabla 2. Análisis para daño tipo piel de cocodrilo

\begin{tabular}{|c|c|c|c|c|}
\hline \multirow{2}{*}{ Tramo } & \multirow{2}{*}{ Ancho (m) } & \multicolumn{2}{|c|}{ Abscisa } & \multirow{2}{*}{ Área tramo $\left(\mathrm{m}^{2}\right)$} \\
\hline & & Desde & Hasta & \\
\hline 1 & 7 & $\mathrm{~K} 0+000,000$ & K0+179,792 & 1258,544 \\
\hline \multicolumn{4}{|c|}{ Área total inspeccionada $\left(\mathrm{m}^{2}\right)$} & 1258,544 \\
\hline \multicolumn{5}{|c|}{ Área total afectada por severidad $\left(\mathrm{m}^{2}\right)$} \\
\hline \multicolumn{5}{|c|}{ Área total afectada por tipo de daño $\left(\mathrm{m}^{2}\right)$} \\
\hline \multicolumn{5}{|c|}{ Peso del daño por severidad dentro del área inspeccionada (\%) } \\
\hline \multicolumn{5}{|c|}{ Peso total por tipo de daño del área inspeccionada (\%) } \\
\hline \multicolumn{5}{|c|}{ PC } \\
\hline \multicolumn{2}{|c|}{ Bajo } & \multicolumn{2}{|c|}{ Medio } & Alto \\
\hline \multicolumn{2}{|c|}{179,71} & \multicolumn{2}{|c|}{6,548} & 0 \\
\hline \multicolumn{2}{|c|}{179,71} & \multicolumn{2}{|c|}{6,548} & 0 \\
\hline \multicolumn{5}{|c|}{186,259} \\
\hline \multicolumn{2}{|c|}{14,279} & \multicolumn{2}{|c|}{0,520} & 0,000 \\
\hline \multicolumn{5}{|c|}{14,800} \\
\hline
\end{tabular}

Fuente: elaboración propia.

El daño que menos se presenta corresponde al grupo de fisura denominada fisuras longitudinales (FL) y transversales (FT) (tabla 3). 
Tabla 3. Daño menos frecuente: Fisuras Transversales (FT)

\begin{tabular}{|c|c|c|}
\hline \multicolumn{3}{|c|}{ FT } \\
\hline Bajo & Medio & Alto \\
\hline 0,169 & 0 & 0 \\
\hline \multicolumn{3}{|c|}{0} \\
\hline 0,169 & $\mathbf{0 , 1 6 9}$ & 0 \\
\hline 0,013 & 0,000 & 0,000 \\
\hline & 0,013 \\
\hline
\end{tabular}

Fuente: elaboración propia.

La información de la media estadística de la muestra evidencia el cambio de velocidad al que son sometidos los articulados cuando se encuentran con un tramo en malas condiciones, ya que es necesaria la desaceleración del articulado. Específicamente, la comparación de la velocidad de los articulados por cada día de la semana en el que fueron tomadas, haciendo la misma comparación de velocidad antes y durante respecto al tramo en buenas condiciones, permitió establecer también que sin importar el día o la hora de la toma de datos el comportamiento de la muestra es el mismo. Con base en esto se puede inferir que, efectivamente, el tramo de pavimento con daño genera el problema en la desaceleración de los articulados, lo cual impacta negativamente el parque automotor.

\section{Conclusiones}

Es prioritario que el tramo de la calzada de la ET-ESMIC sea sometido a actividades de mantenimiento de pavimentos de las dos categorías que se aplican: preventivas y correctivas. El mantenimiento preventivo tiene el propósito de proteger el pavimento y reducir su rata de deterioro, mientras que el mantenimiento correctivo se hace con el fin de corregir fallas específicas del pavimento o áreas deterioradas, para lo cual se puede emplear toda la planificación del Manual de Mantenimiento y Rehabilitación de Pavimentos Flexible (Universidad Nacional de Colombia e Instituto Nacional de Vías [Invías], 2006).

\section{Agradecimientos}

Los autores agradecen a la Escuela Militar de Cadetes “General José María Córdova”.

\section{Declaración de divulgación}

Los autores declaran que no existe ningún potencial conflicto de interés relacionado con el texto.

\section{Financiamiento}

Los autores no declaran fuente de financiamiento para la realización de este artículo.

\section{Sobre los autores}

Johan Camilo Rodríguez Cerón es estudiante de la Facultad de Ingeniería Civil de la Escuela Militar de Cadetes “General José María Córdova”, Bogotá, D. C., Colombia.

Sergio Esteban Valero Hernández es estudiante de la Facultad de Ingeniería Civil de la Escuela Militar de Cadetes “General José María Córdova”, Bogotá, D. C., Colombia. 
Javier Alejandro Villota Arteaga es estudiante de la Facultad de Ingeniería Civil de la Escuela Militar de Cadetes “General José María Córdova”, Bogotá, D. C., Colombia.

Octavio Vega es ingeniero civil de la Universidad Minuto de Dios, especialista en diseño construcción y conservación de vías de la Escuela Colombiana de Ingeniería Julio Garavito. Docente en la Facultad de Ingeniería Civil de la Escuela Militar de Cadetes "General José María Córdova”, Bogotá, D. C., Colombia.

Johanna Carolina Ruiz Acero. Es Ingeniera Civil de la Universidad Militar Nueva Granada. Magíster en Geotecnia de la Pontificia Universidad Javeriana. Docente en la Facultad de Ingeniería Civil, Facultad de Ingeniería Civil de la Escuela Militar de Cadetes "General José María Córdova”, Bogotá, D. C., Colombia. Contacto: johanna.ruiz@esmic.edu.co

\section{Referencias}

Beltrán, L. (septiembre de 2016). Las fallas de los pavimentos de Transmilenio. UN Periódico, 57. Recuperado de http://historico.unperiodico.unal. edu.co/ediciones/57/06.htm

II compendio de buenas prácticas en gestión vial descentralizada. (2015). Recuperado de http://www. proviasdes.gob.pe/publicaciones/gestion_vial_ desc/IIConcursoBuenasPracticaGVD_Compendio.pdf

Universidad Nacional de Colombia e Instituto Nacional de Vías [Invías]. (2006). Estudio e investigación del estado de actual de las obras de la red nacional de carreteras: Manual para la inspección visual de pavimentos flexibles [documento en línea]. Recuperado de https://www. invias.gov.co/index.php/archivo-y-documentos/documentos-tecnicos/manuales-de-inspeccion-de-obras/664-manual-para-la-inspeccion-visual-de-pavimentos-rigidos/file 